\title{
Traduire
}

Ine autre perspective sur la tataduction

Revue française de la traduction

$244 \mid 2021$

Des jeux et des mots

\section{Au cœur de la terminologie du jeu vidéo. L'absence de ressources, frein majeur pour les traducteurs}

Sarah Theroine, María Isabel Rivas Ginel et Aurélie Perrin

\section{(2) OpenEdition}

Édition électronique

URL : https://journals.openedition.org/traduire/2285

DOI : 10.4000/traduire.2285

ISSN : 2272-9992

Éditeur

Société française des traducteurs

Édition imprimée

Date de publication : 15 juin 2021

Pagination : $27-40$

ISSN : 0395-773X

\section{Référence électronique}

Sarah Theroine, María Isabel Rivas Ginel et Aurélie Perrin, « Au cœur de la terminologie du jeu vidéo. L'absence de ressources, frein majeur pour les traducteurs », Traduire [En ligne], 244 | 2021, mis en ligne le 15 juin 2021, consulté le 01 juillet 2021. URL : http://journals.openedition.org/traduire/2285 ; DOI : https://doi.org/10.4000/traduire.2285 


\section{Au coeur de la terminologie du jeu vidéo}

L'absence de ressources, frein majeur pour les traducteurs

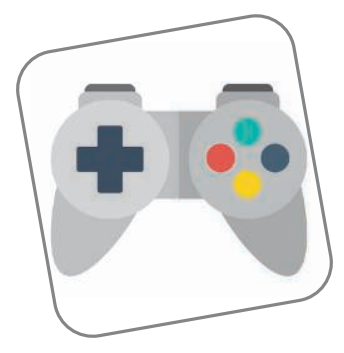

Sarah Theroine, María Isabel Rivas Ginel, Aurélie Perrin

\section{Technologies et gestion terminologique: une étude auprès des utilisateurs}

L'industrie vidéoludique est un secteur d'activité dont la croissance n'a cessé de s'amplifier tant sur le plan économique que du point de vue professionnel depuis sa naissance dans les années 1970. Une étude publiée sur le site spécialisé Newzoo.com dévoile que le chiffre d'affaires du secteur a atteint les 174,9 milliards de dollars en 2020, dépassant ainsi, une fois de plus, celui de l'industrie cinématographique. À l'intérieur de cette industrie, la localisation revêt une importance particulière, car le traducteur doit sans relâche se consacrer à un travail d'adaptation culturelle du contenu, au niveau non textuel (unité de mesure, monnaies, lois) et culturel (références, jeux de mots, etc.). Toutefois, le traducteur de jeux vidéo est également confronté à des épiphénomènes typiques de ce secteur, comme les variables et balises, ou un 
processus de traduction que Miguel Á. Bernal-Merino (BernalMerino, 2015: 81) décrit comme un double-blind process caractérisé par une scission entre image et texte et par un manque de linéarité du texte. En effet, une enquête récemment menée auprès de traducteurs de jeux vidéo (Rivas Ginel, 2020) montre que, si $76,61 \%$ des sondés signalent avoir accès à des documents de référence, seuls 29,68\% d'entre eux ont accès à l'environnement visuel du jeu.

Une autre difficulté à laquelle font face les traducteurs est liée à la complexité grandissante du contenu. Non seulement les jeux modernes combinent l'écrit et l'oral avec des images en mouvement, mais ils requièrent en outre la maîtrise d'un vocabulaire toujours plus spécifique aux différentes souscatégories, rejoignant ainsi des problématiques connues en traduction spécialisée, mais que les jeux vidéo éclairent d'un jour nouveau. Par conséquent, la localisation des jeux vidéo oblige les développeurs à prévoir plusieurs phases de test après la traduction proprement dite afin de s'assurer que le contenu est correctement intégré aux supports concernés, et de pouvoir régler les bugs qui peuvent apparaître, dus, essentiellement, à cette scission caractéristique du secteur. La figure suivante, provenant d'une enquête adressée spécifiquement aux testeurs linguistiques de jeux vidéo, montre la fréquence des différents types de bugs par ordre de prévalence:

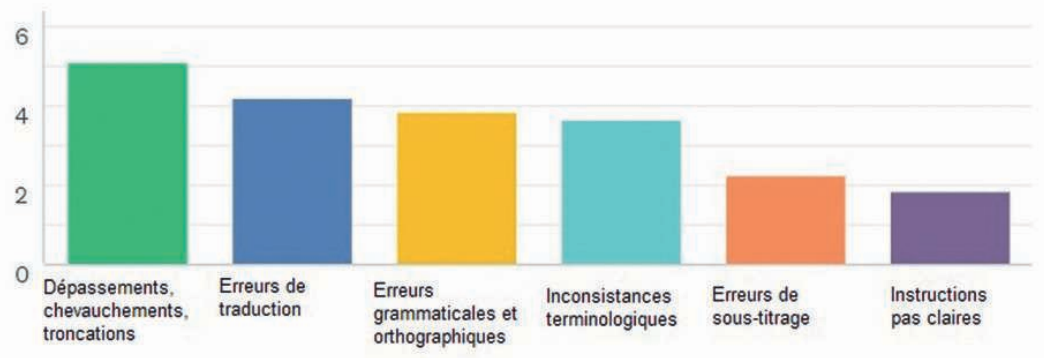

Figure 1: Prévalence de types de bugs.

Lorsqu'il leur a été demandé d'identifier quelles étaient les causes possibles de ces incohérences terminologiques, ils ont signalé pour $33,27 \%$ le manque de ressources (glossaires ou character bibles), suivi pour 24,18\% par l'option regroupant le manque de ressources, le travail réalisé par un trop 
grand nombre de traducteurs et le manque d'instructions reçues. Nous allons analyser brièvement des solutions technologiques disponibles actuellement, avant d'attaquer le cœur du problème: l'absence de ressources.

Le marché propose un large éventail d'outils: correcteurs orthographiques, gestionnaires de terminologie, logiciels de traduction assistée par ordinateur (TAO), logiciels de traduction automatique (TA), logiciels de concordance ou encore compilateurs de corpus. Des études antérieures dans le domaine de l'adoption des nouvelles technologies (Lagoudaki, 2006 ; Zaretskaya Ana et al., 2017) montrent que si les traducteurs préfèrent travailler avec des outils disposant de multiples fonctionnalités, ces systèmes, davantage axés sur la technologie que sur le confort de l'utilisateur, sont extrêmement complexes. Parmi les logiciels de gestion et d'extraction terminologique, nous trouvons différents outils qui peuvent être soit indépendants soit intégrés dans des logiciels TAO ou de compilation de corpus. En analysant les réponses à l'enquête, nous constatons que, bien que les traducteurs interrogés soient au courant de l'existence de ce genre d'outils, 64,35\% ne les utilisent pas, et seulement $6,29 \%$ signalent en faire usage de façon régulière. La figure 2 montre les réponses à la question sur l'usage des systèmes autonomes:

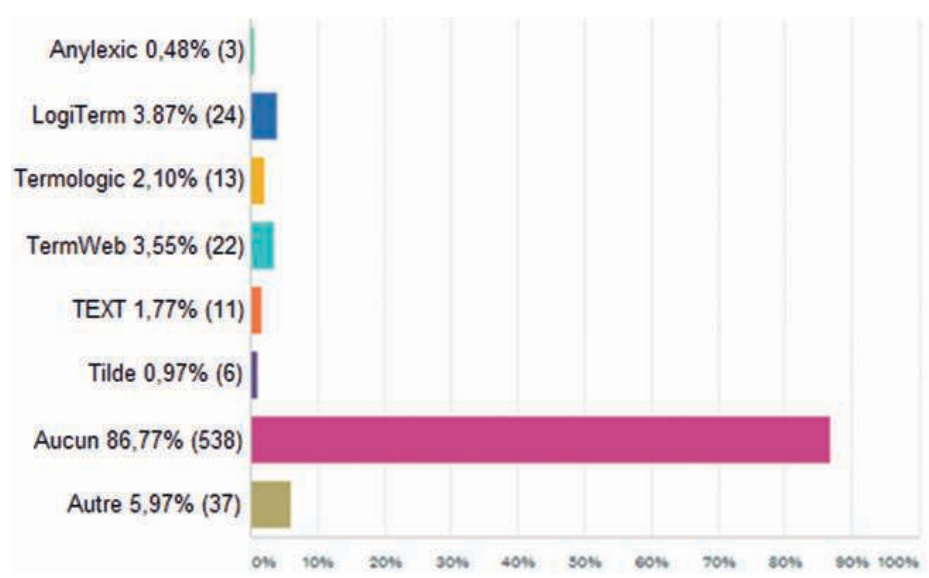

Figure 2: Utilisation des systèmes autonomes d'extraction et gestion terminologique. 
Dans la catégorie "autre» apparaissent SynchroTerm, Term Morphology Editor, TemExtract, mais surtout beaucoup de réponses qui montrent que les professionnels utilisent exclusivement les systèmes intégrés dans leur logiciel TAO (23 sur 37). Le tableau numéro 1 ci-dessous montre les résultats à la question sur le recours à l'extraction et la gestion terminologique à travers les fonctionnalités intégrées dans des gestionnaires de mémoire de traduction:

\begin{tabular}{|c|c|}
\hline OUTIL TAO & NOMBRE DE RÉPONSES \\
\hline SDL MultiTerm Extract & 173 \\
\hline qTerm $^{\text {TM }}$ & 49 \\
\hline QuickTerm & 24 \\
\hline crossTerm & 7 \\
\hline Memsource & 3 \\
\hline Crowding & 1 \\
\hline Wordfast & 1 \\
\hline Lokalise & 1 \\
\hline DejaVu & 1 \\
\hline Aucun & 393 \\
\hline
\end{tabular}

Tableau 1 : Recours à l'extraction et la gestion terminologique intégrée dans des outils TAO.

L'enquête montre aussi que les outils de gestion terminologique de corpus sont les moins utilisés ( $94,19 \%$ n'utilisent aucun outil) et que seulement 53 participants (soit 8,39\%) compilent leur propre corpus. Ces données sont dues, principalement, à l'absence de ressources terminologiques certifiées et officielles, un frein majeur pour les traducteurs de jeux vidéo.

\section{Les ressources terminologiques: accès et fiabilité}

Les ressources terminologiques les plus fréquemment utilisées dans un projet de localisation de jeu vidéo peuvent 
être classées en deux catégories: les ressources propres au jeu et celles appartenant aux plateformes de publication. La première catégorie englobe les documents transmis par les développeurs dans le kit de localisation, comprenant guide de style, glossaire de termes à traduire ou non, captures d'écran ou encore documents de travail sur l'univers du jeu, les personnages ou même le déroulement du jeu. Cependant, comme le montre l'enquête menée auprès de traducteurs de jeux vidéo (Rivas Ginel, 2020), près de $25 \%$ des traducteurs n'ont pas accès à ces documents, souvent pour des raisons de haut degré de confidentialité, de retards ou de changements majeurs dans le développement du jeu.

Si l'accès aux ressources terminologiques propres aux jeux dépend finalement des contenus que les développeurs peuvent partager et, dans une certaine mesure, des contenus mis à disposition par les communautés de joueurs, l'accès aux ressources officielles est plus complexe. Celles employées par les plateformes (Windows, PlayStation, Xbox, Nintendo Switch) ou encore par les jeux mobiles (Android, iOS), appartenant à la deuxième catégorie, sont des éléments clés du processus de certification obligatoire, dans la mesure où seule cette dernière garantit un usage adéquat des termes avant leur publication sur les différents systèmes. Néanmoins, la question des droits d'accès des traducteurs aux matériaux linguistiques de certaines plateformes ou éditeurs perdure. En effet, seule la terminologie officielle de Microsoft reste relativement facile d'accès, grâce au portail linguistique mis en ligne par la multinationale. Le manque de matériaux linguistiques a des conséquences notables et impacte les différents acteurs du processus, jusqu'à la phase du test de localisation (LQA).

En dépit de l'importance des ressources précédemment citées pour assurer la publication d'un jeu et éviter d'importants coûts de correction des erreurs de conformité repérées à l'étape de la LQA ou, pire encore, de la certification, elles ne sont pas toujours fournies aux traducteurs, alors qu'elles leur sont indispensables. II faut cependant noter que cette tendance évolue et que les clients, développeurs ou agences, sont de plus en plus nombreux à transmettre des glossaires officiels complets ou relativement complets au format Excel ou intégrés dans les outils de TAO. Malheureusement, ces 
derniers restent peu nombreux et cette pratique est très variable selon l'expérience en localisation du studio de développement ou de l'agence. À défaut d'avoir accès à des glossaires structurés, il reste possible d'accéder à des sites officiels dédiés aux utilisateurs afin de télécharger les notices de leur équipement. À noter que souvent, la version du manuel étant directement intégrée aux casques $V R$, l'extraction des données terminologiques est rendue extrêmement compliquée.

Il existe cependant des ressources non officielles qu'il est possible d'exploiter dans une certaine mesure. Elles sont créées bénévolement par la communauté (wikis, forums ou sites) afin d'aider les futurs joveurs. Ces contenus se présentent sous la forme de sites multilingues, les wikis par exemple, ou de sites monolingues spécialisés destinés à un public de joveurs dans une langue précise. Les ressources multilingues sont développées, maintenues et alimentées par des joveurs maîtrisant différentes langues et plus ou moins organisées selon les communautés. Les sites monolingues ont plutôt tendance soit à se concentrer sur certains aspects précis d'un jeu, soit à être les plus exhaustifs possibles afin d'informer une communauté plus vaste. Dans les deux cas, la disponibilité de ces contenus en plusieurs langues est tributaire de l'engagement des membres de ces communautés. La terminologie liée aux jeux constitue un point d'appui pour employer le vocabulaire que les joveurs utilisent déjà entre eux, même si les termes sont souvent dérivés de l'anglais, car de nombreux jeux sortent en "accès anticipé » ou en «version bêta» avant même leur localisation.

Afin de pallier ces problèmes terminologiques, le marché propose des outils tels que les logiciels ou sites de création de corpus linguistiques comme Sketch Engine, SKELL, KWICFinder ou encore WebCorp qui permettent aux utilisateurs de créer des corpus spécialisés en recensant et en compilant des ressources disponibles sur internet traitant du domaine de spécialité dans une langue ciblée. Le fonctionnement de ces outils est similaire à un moteur de recherche dans lequel l'utilisateur saisit des mots clés pertinents dans son domaine de spécialité. Les outils compilent ensuite tous les termes en référence, appelés "concordances», en lien avec ces mots clés trouvés sur des sites spécialisés, articles 
ou livres disponibles sur internet. Néanmoins, il est essentiel d'avoir les compétences et connaissances nécessaires à leur utilisation, ainsi que l'accès aux ressources de la langue de spécialité définie pour pouvoir les exploiter, ce qui n'est pas toujours le cas de la terminologie du jeu vidéo. Il est facile, lors de la création d'un corpus en français portant sur la terminologie du jeu vidéo, de corréler les données fournies précédemment. En effet, une recherche de mots clés tels que: "lexique du jeu vidéo», "vocabulaire du jeu vidéo», "terminologie du jeu vidéo» ou encore "vocabulaire gaming» ne donne que peu de ressources (seize liens seulement, dont sept renvoyant à Wikipédia lexique du jeu vidéo et $M M O R G$ ). De plus, lors de l'extraction des termes par le logiciel, on remarque la prédominance de la langue anglaise dans les termes recensés. À l'inverse, si l'on essaie de créer un corpus en anglais avec les mots clés gaming dictionary, gaming terminology, glossary of video game terms et gaming lexicon, il y a sensiblement plus de sections terminologiques (glossaires, lexiques, dictionnaires), puisque la recherche débouche sur 55 liens.

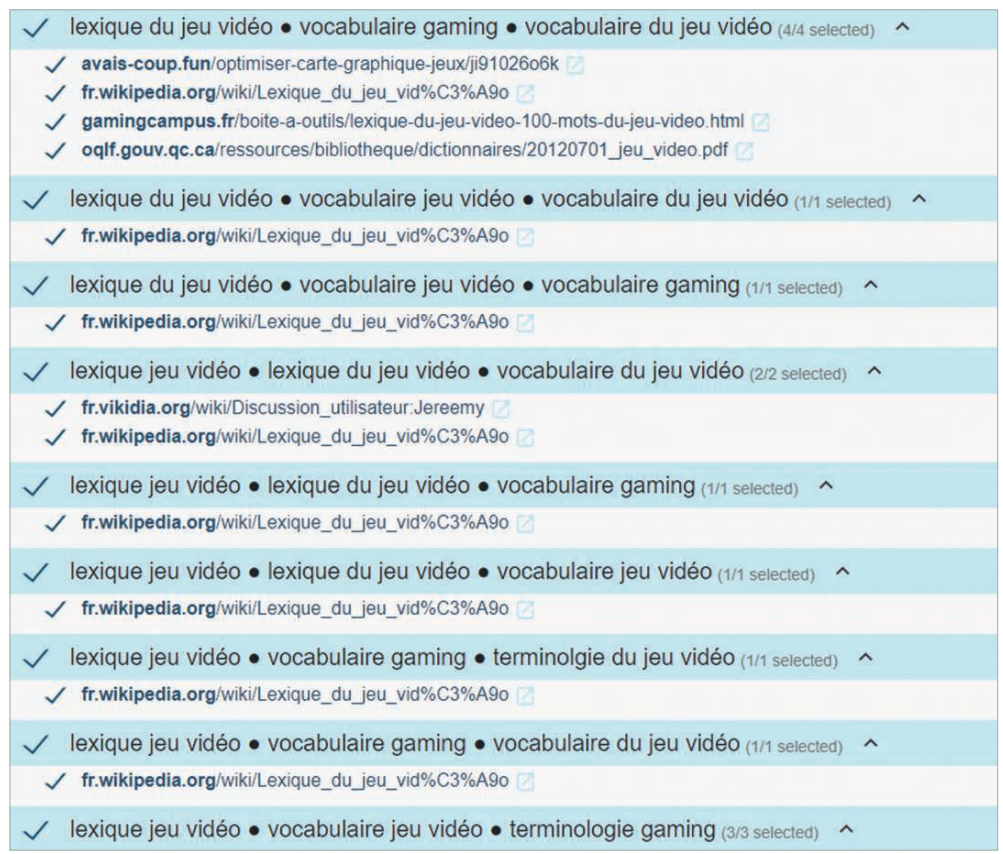

Figure 3: Résultat d'une compilation des sources suivant les mots clés français. 
En élargissant notre analyse des termes anglais et francais extraits par le logiciel, on peut remarquer deux éléments importants. Tout d'abord, le vocabulaire recensé ne correspond pas au vocabulaire utilisé par les traducteurs professionnels, mais à celui employé par les joueurs. Deux problématiques peuvent découler de cette première observation: si les termes appartiennent au domaine du jeu vidéo, font-ils réellement partie du vocabulaire nécessaire aux professionnels de la traduction? Peuvent-ils être considérés par les traducteurs comme appartenant à la langue de spécialité vidéoludique? De plus, bien que les définitions données par les faibles ressources rencontrées soient dans les langues cibles pour chaque corpus monolingue correspondant, aucune équivalence terminologique française n'est proposée.

De ce fait, cette analyse terminologique menée via la création d'un corpus ne peut que corroborer les informations et les problèmes de fiabilité et de disponibilité des ressources, énumérés plus tôt, auxquels font face les traducteurs du jeu vidéo. Cependant, cette recherche laisse aussi supposer que la langue de spécialité, étant encore en pleine évolution, manque elle-même de ressources. En ce sens, on pourrait qualifier ce domaine de specialized narrow domain (Skadina, Vasiljevs, Skadils et al., 2010), autrement dit, de domaine de spécialité étroit dû à sa technicité et à sa grande diversité.

\section{Le néologisme comme mécanisme compensatoire}

La terminologie du jeu vidéo peut emprunter, en fonction du genre du jeu, des termes spécifiques à ces domaines de spécialité. En ce sens, elle peut être considérée comme étant pluriculturelle (Leroyer \& Bergenholtz, 2013: 168). En effet, les jeux compétitifs en équipe ont la particularité d'employer un jargon hautement spécialisé et très technique dont l'unique but est de répondre à un besoin précis: communiquer avec n'importe quel joveur à travers le monde afin d'élaborer des stratégies. Ainsi sont apparus des termes tels que gank ou ganker (néologisme né de Gang Kill) ou le rôle de jungler (joveur dont le rôle est de «sécuriser» une zone précise, à savoir la jungle dans League of Legends). Si la Commission 
générale de terminologie et de néologie (CGTN) s'emploie à proposer des équivalents en français de la terminologie issue des jeux vidéo, ceux-ci se heurtent bien souvent à un public qui n'hésite pas à exprimer sa réticence, notamment à propos de termes comme hardcore gamer auquel il est recommandé de préférer "hyperjoveur».

II revient aux traducteurs de faire un choix parfois épineux quant aux termes qu'ils doivent utiliser. Selon une règle tacite, l'emploi des néologismes est recommandé lorsque l'on s'adresse à un public évoluant dans certains types de jeux compétitifs ou utilisant un vocabulaire très précis; a contrario, l'emploi de la nouvelle terminologie française est privilégié pour des jeux plutôt destinés au grand public. Évidemment, cela dépend de plusieurs paramètres, tels que la décision du client dans la manière de s'adresser aux joueurs ou l'expertise des traducteurs à l'heure de déterminer le vocabulaire à employer par rapport au public visé. Comme nous l'avons déjà signalé, l'accès aux ressources repose sur la capacité des développeurs à fournir un kit de localisation aussi complet que possible et contenant également les glossaires officiels des différentes plateformes sur lesquelles leur jeu sera publié, ce qui n'est pas toujours le cas. Ainsi, certains traducteurs ont compilé des glossaires (rendus publics ou à usage privé) compatibles avec les outils de TAO les plus courants. Cependant, il n'est pas toujours possible d'intégrer ces glossaires aux projets lorsque le travail est effectué avec des outils conçus sur mesure ou lorsque la configuration du projet ne permet tout simplement pas d'ajouter d'autres sources. Du côté des agences, l'intégration des glossaires dans les outils de TAO est primordiale puisque de nombreux traducteurs n'utilisent pas d'outils autonomes. II serait aussi impératif de créer une banque de données consultable et actualisable regroupant les ressources officielles afin de limiter le nombre d'erreurs terminologiques d'ordre général.

\section{Création d'un dictionnaire multilingue, accessible et collaboratif}

Afin de compenser la pénurie de ressources terminologiques dans le domaine du jeu vidéo, un projet de création de dictionnaire multilingue est en cours, prenant l'anglais comme 
langue source et l'espagnol et le français comme langues cibles (Linossier \& Theroine, 2020). En effet, du point de vue de la communication, l'objectif est non seulement de combler les lacunes lexicographiques des traducteurs, correcteurs et réviseurs de jeux vidéo, mais aussi de résoudre les problèmes de réception du texte en langue cible et ceux liés à la localisation et à la LQA. En parallèle, l'ouvrage offre aussi des fonctions cognitives en fournissant des informations encyclopédiques, culturelles et des données spécialisées dans le domaine vidéoludique (Leroyer \& Bergenholtz, 2013: 160). L'élaboration de ce projet repose sur la base d'un lexique préexistant anglais-espagnol qui a été retravaillé en amont afin de favoriser l'ajout de termes plus techniques et plus pertinents (interfaces, paramètres et crédits).

La structure et le contenu du dictionnaire s'appuient sur les différents types de données lexicographiques nécessaires à la bonne efficience du projet (Fuertes-Olivera \& Tarp, 2014). De ce fait, les utilisateurs pourront trouver, classé par ordre alphabétique dans la langue source (anglais), chaque terme auquel s'ajouteront les données suivantes:

- un équivalent français du terme en précisant le genre, le nombre et la classe grammaticale à laquelle il appartient. À noter que s'il n'existe aucun équivalent français, une proposition terminologique sera présentée. La décision d'employer cette dernière ou de lui préférer le terme anglais ou un autre terme reviendra au traducteur qui jugera au mieux quelle stratégie employer en fonction du contexte;

- diverses définitions avec mention de leurs sources;

- ses abréviations ou acronymes, le cas échéant;

- des précisions spécifiant le contexte situationnel, si nécessaire;

- les synonymes et les préférences d'utilisation dans la langue de spécialité afin de guider l'utilisateur vers le terme le plus pertinent à employer en fonction du contexte et du terme source;

- des exemples d'utilisation illustrant l'emploi du terme au sein du domaine de spécialité en question;

- des collocations pouvant être des noms, des verbes, des adjectifs et des phrases courtes fréquemment associés et/ou utilisés conjointement au terme. 


\begin{tabular}{|c|c|c|c|c|c|c|c|c|}
\hline & 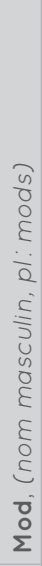 & 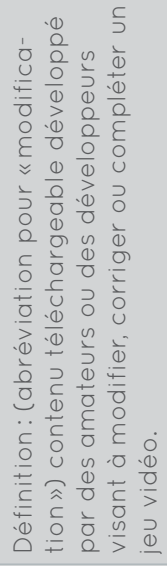 & 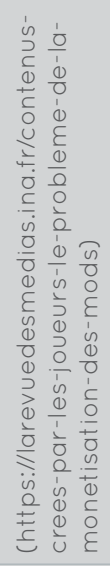 & 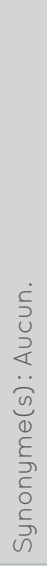 & 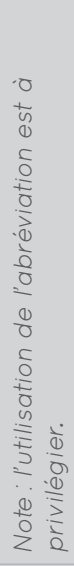 & 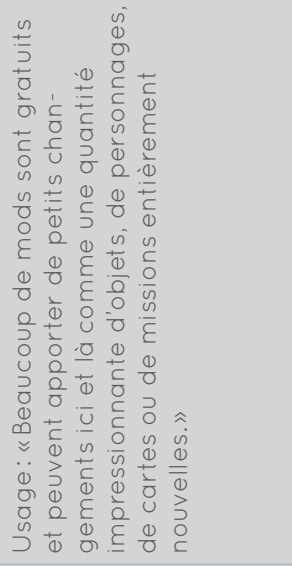 & 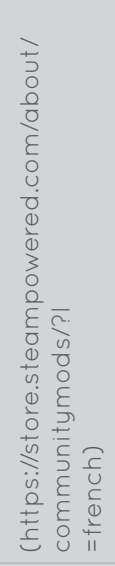 & 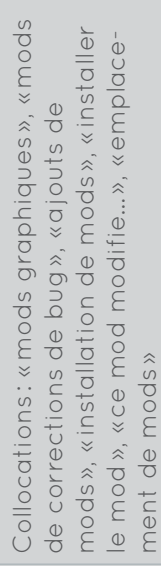 \\
\hline & 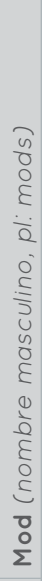 & 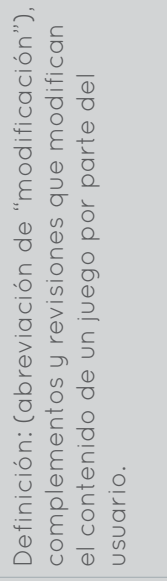 & 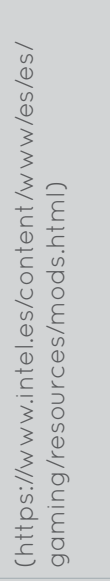 & 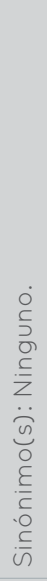 & 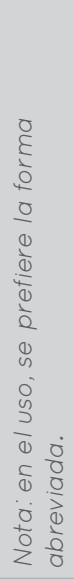 & 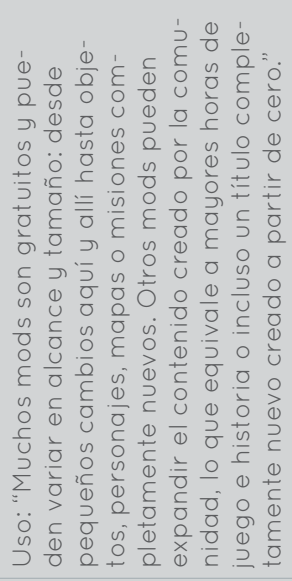 & 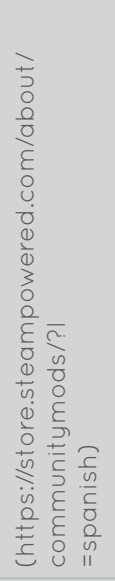 & 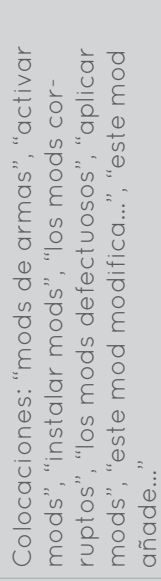 \\
\hline 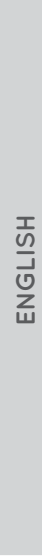 & $\begin{array}{l}n \\
0 \\
0 \\
\varepsilon \\
\varepsilon \\
\bar{Q} \\
5 \\
5 \\
0 \\
5 \\
0 \\
0 \\
0\end{array}$ & 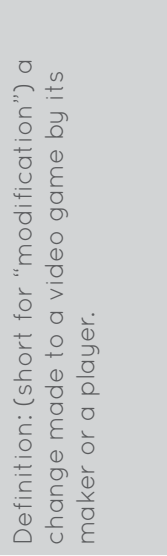 & 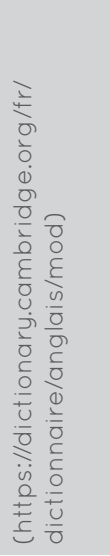 & & $\begin{array}{l}0 \\
0 \\
0 \\
0 \\
0 \\
0 \\
0 \\
0 \\
0 \\
0 \\
5 \\
\vdots \\
0 \\
\vdots \\
\pm \\
0 \\
\frac{1}{0} \\
0 \\
0 \\
+ \\
0 \\
0 \\
0 \\
5 \\
5 \\
0\end{array}$ & 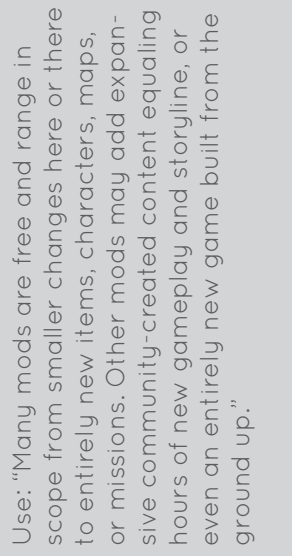 & 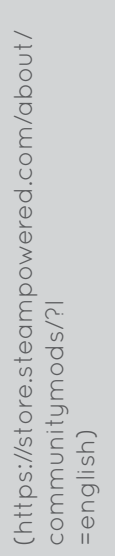 & 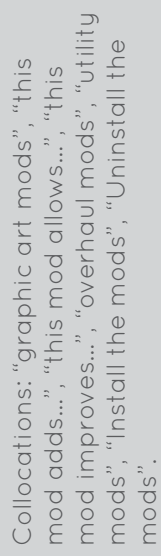 \\
\hline
\end{tabular}

$\frac{0}{2}$ 
Bien que le projet ne soit pas encore achevé, certaines questions peuvent d'ores et déjà être soulevées: l'exploitation de la terminologie propre aux plateformes nécessite-t-elle un accord bipartite pour les faire figurer dans cette ressource? La terminologie du jeu vidéo est-elle évolutive ou statique? Car ce sont bien les problématiques majeures de ce travail qui ont conduit à élargir les objectifs du projet. En effet, il n'est plus seulement question de fournir aux utilisateurs une ressource terminologique fiable, mais de pouvoir la modifier ou l'actualiser en cas d'évolution ou d'enrichissement de la terminologie. En plus d'être accessible au public, elle doit être rendue exploitable et compatible avec les logiciels de traduction, ainsi que les outils de gestion de corpus.

\section{theroine.sarah89@gmail.com isabelrivasginel@gmail.com aurelie@orelitranslation.com}

Sarah Theroine, étudiante diplômée du master T2M (traduction multimédia) de l'université de Bourgogne et future doctorante CIFRE en cotutelle avec les laboratoires TIL et Informatique de l'université de Bourgogne. Ses recherches porteront sur l'amélioration de l'idiomaticité d'assistant conversationnel à travers la notion de patterns.

María Isabel Rivas Ginel, ATER d'espagnol, anglais et français à I'ESIT et doctorante en sciences du langage en cotutelle à l'université de Bourgogne Franche-Comté et l'université de Valladolid. Ses recherches portent sur la traduction audiovisuelle et la localisation de jeux vidéo.

Aurélie Perrin, traductrice anglais-français spécialisée en localisation de jeux vidéo intervenant dans ce domaine au sein du master T2M (université de Bourgogne Franche-Comté) et animatrice d'une émission Twitch destinée à promouvoir les métiers de l'industrie vidéoludique, dont la localisation. 
BERNAL-MERINO Miguel Ángel, Translation and Localisation in Video Games: Making Entertainment Software Global, New York, Routledge, Routledge Advances in Translation and Interpreting Studies, 2015.

FUERTES-OLIVERA Pedro et TARP Sven, Theory and Practice of Specialised Online Dictionaries. Lexicography versus Terminography, Berlin, DE GRUTYER, Lexicographica Series Maior, volume 146, 2014.

LAGOUDAKI Elina, Translation Memories Survey 2006: Users' perceptions around TM use., Londres, Proceedings of the ASLIB International Conference Translating \& the Computer, 2006.

LÉGIFRANCE, Vocabulaire de la culture et des médias (liste de termes, expressions et définitions adoptés), JORF n0084 du 8 avril 2017, https://www.legifrance.gouv.fr/ jorf/id/JORFTEXTO00034391219, consulté le 06/03/2021.

LEROYER Patrick et BERGENHOLTZ Henning, «Métalexicographie culturelle, fonctions lexicographiques et finalité pragmatique», in Études de linguistique appliquée, CAIRN, Danemark, 2013, p. 160-168.

LEROYER Patrick, «La correction de la traduction L2 > L1 comme fonction du dictionnaire spécialisé», in F. Maniez, P. Durin, N. Arlin, et C. Rougement (dir.), Corpus et dictionnaires de langues de spécialité, Grenoble, PUG, travaux du CRTT, 2008, p. 111-133.

LEROYER Patrick, The Forms and Functions of Specialised Dictionaries for Practical Purposes, in K. Akasu, S. Uchida (dir.), Proceedings of the 7th Asialex International Conference on Lexicography: Theoretical and Practical Perspectives, Kyoto, Asialex, 2011, p. 313-322.

LINOSSIER Noëllie et THEROINE Sarah, Building up a multilingual dictionary, université de Bourgogne Franche-Comté, Dijon, France, Language and Culture in Videogames, 2020.

MICROSOFT, Portail linguistique, https://www.microsoft.com/fr-fr/language, consulté le 06/03/2021.

NEWZOO, Key Numbers, https://newzoo.com/key-numbers/, consultéle 11/02/2021.

PROGRESSER SUR LOL, Lexique League of Legends, 2015, https://www.progressersurleagueoflegends.fr/guides/guides-connaissances/lexique-league-of-legends/, consulté le 06/03/2021

RIVAS GINEL María Isabel, Ergonomics of CAT tool usage for game localisation: a survey for game testers and localisers, France, Language and Culture in Videogames, 2020.

SKADILA Inguna et al., "Analysis and Evaluation of Comparable Corpora for Under-Resourced Areas of Machine Translation", in Proceedings of the 3rd Workshop on Building and Using Comparable Corpora, European Language Resources Association (ELRA), La Valletta, Malta, 2010. 
SUIRAM, Oubliez «hardcore gamer», il faut dire «hyper joueur » maintenant..., HITEK.fr, https://hitek.fr/actualite/on-nous-empeche-de-dire-hardcoregamer_12614, consulté le 06/03/2021.

ZARETSKAYA Anna et al., «User Perspective on Translation Tools: Findings of a User Survey», in CORPAS PASTOR Gloria et DURÁN-MUÑOZ (dir.) Isabel Durán-Muñoz, Trends in E-Tools and Resources for Translators and Interpreters, Leiden/Boston, Brill, 2017, p. 37-56. 